\author{
Brian CRAIN ${ }^{1}$ \\ Shiyin YAO ${ }^{1}$ \\ Vina KEOPHILAONE ${ }^{1}$ \\ Victor PROMESSI ${ }^{1}$ \\ McNancy KANG ${ }^{1}$ \\ Alcide BARBERIS ${ }^{2,3}$ \\ Roberto MA.J 2 \\ Emanuela MURA ${ }^{2}$ \\ Nadia PASSINI ${ }^{2}$ \\ Johanna HOLLDACK ${ }^{2}$ \\ Ricardo OCHOA ${ }^{4}$ \\ Howard B. COTTAM ${ }^{1}$ \\ Dennis A. CARSON ${ }^{1}$ \\ Tomoko HAYASHI ${ }^{1}$ \\ ${ }^{1}$ Moores Cancer Center, \\ University California San Diego, \\ La Jolla CA, \\ USA. 92093-0695 \\ 2 Telormedix SA, \\ Via della Posta 10 \\ CH-6934 Bioggio, \\ Switzerland \\ ${ }^{3}$ Humabs BioMed SA \\ CH-6500 Bellinzona, \\ Switzerland \\ ${ }^{4}$ Pre-Clinical Safety Inc. \\ P.O. Box 718 Niantic, \\ CT 06357
}

Reprints: Tomoko Hayashi

<thayashi@ucsd.edu>

Article accepted on 6/28/2013

\section{Inhibition of keratinocyte proliferation by phospholipid-conjugates of a TLR7 ligand in a Myc-induced hyperplastic actinic keratosis model in the absence of systemic side effects}

\begin{abstract}
Background. The Toll-like receptor 7 (TLR7) activator imiquimod (IMQ) is safe and effective in treating actinic keratosis; however, an intermittent treatment regimen is necessary because of excessive local reactions. Objectives. To evaluate in vitro potency, pharmacodynamics/pharmacokinetics, toxicity and efficacy in vivo of the newly developed TLR7 ligand-phospholipid conjugate, TMX-202, in a gel formulation. Material and Methods. The effects of TMX-202 were assessed both in vitro on a murine macrophage cell line and in primary bone marrow-derived dendritic cells and in vivo on mice (C57BL/6-wild type, Myd $88^{-/}$and $\left.T l r 7^{-/}\right)$. Results. TMX-202 was more potent than IMQ in vitro using murine and human cells. In contrast, in vivo it showed less systemic pro-inflammatory activity and better safety than IMQ. Moreover, the TMX-202 gel formulation exhibited at least comparable efficacy to Aldara in a mouse model for skin proliferative diseases. Conclusion. TMX-202 is safe and efficacious without causing excessive adverse effects, suggesting that it may be an alternative to Aldara for the treatment of proliferative skin conditions.
\end{abstract}

Key words: Toll like receptor-7, skin proliferative disease, Myc
A

ldara, a 5\% cream formulation of imiquimod (IMQ), is approved for external genital warts, superficial basal cell carcinoma and actinic keratosis $(\mathrm{AK})[1,2]$. IMQ is a synthetic small molecule ligand that triggers the activation of innate immune cells by stimulating Toll-like receptors 7 (TLR7) and 8 (TLR8). IMQ preferentially activates TLR7, while its agonistic activity toward TLR8 appears to be much weaker [3]. TLR7 is located intracellularly in the endosomal compartments of innate immune cells, such as myeloid and plasmacytoid dendritic cells, monocytes and mast cells [4]. Under certain conditions, it is also expressed on $\mathrm{T}$ and $\mathrm{B}$ lymphocytes or natural killer cells [5]. Signaling through TLR7/8 activates the NF- $\kappa \mathrm{B}$ dependent secretion of pro-inflammatory cytokines. A recent report indicated that TLR-independent activation by IMQ through adenosine receptors or by inflammasome activation is also involved in the mechanism of action of IMQ in skin proliferative disorders [1].

Our laboratory evaluated 9-benzyl-8-hydroxy-2-(2methoxyethoxy) adenine (SM360320; designated here as 1V136), which has been shown to be a specific TLR7 ligand [6]. We further synthesized various derivatives of 1V136 conjugated to proteins, lipids or polyethylene glycol [7]. The conjugations improved the immune stimulatory potency in vitro and reduced non-specific toxicity in vivo [7, 8]. TMX-202 was prepared similarly, by conjugating the TLR7 ligand to a $\mathrm{C}-12$ phospholipid via a versatile benzoic acid functional group [7].

Although Aldara is generally well-tolerated, clinical studies indicate that Aldara can induce systemic as well as local adverse effects $[9,10]$. The systemic adverse effects, including fever, arthralgia, headache, myalgia and lymphadenopathy, are caused by the absorption of IMQ through the skin, followed by pro-inflammatory cytokine release in the circulation [11]. A local IMQ-induced cytokine dermatitis may enhance the systemic absorption of the drug and increase the incidence of systemic adverse effects $[10,11]$. We have reported that phospholipid conjugates of TLR7 ligands retained in vitro potency without causing high levels of acute cytokine release in vivo [7]. Indeed, one TLR7 ligand-phospholipid conjugate was more effective in inhibiting melanoma growth than the parent compound 
[8]. In this study, the newly developed TLR7 ligandphospholipid conjugate, TMX-202, in a gel formulation, was evaluated for in vitro potency, pharmacodynamics (PD)/pharmacokinetics (PK), toxicity and efficacy in vivo. The results indicate that TMX-202 was more potent than IMQ in vitro using murine and human cells. In contrast, in vivo it showed less systemic pro-inflammatory activity and better safety than IMQ. Moreover, the TMX-202 gel formulation exhibited at least comparable efficacy to Aldara in a mouse model for skin proliferative diseases.

\section{Materials and methods}

\begin{abstract}
Animals
Seven- to 9-week-old C57BL/6 (wild type) mice were purchased from the Jackson Laboratories (Bar Harbor, MA). $M y d 88^{-/-}$and $T l r 7^{-/-}$mice were a gift from Dr. Shizuo Akira (Osaka University). Inv-MycERTAM transgenic mice (InvMycER mice) were obtained from Drs. Michael Khan and Stella Pelengaris (University of Warwick, UK) [12]. All strains except wild type mice were bred at the University of California, San Diego (UCSD). Bone marrow chimeras were generated by injecting $10^{7}$ bone marrow cells intravenously into whole body irradiated $(9.5 \mathrm{~Gy})$ recipient mice [13]. The UCSD Institutional Animal Care and Use Committee approved all animal experiments.
\end{abstract}

\section{Reagents}

Tamoxifen (Sigma Chemical Co. St Louis, LA) was dissolved in ethanol at $5 \mathrm{mg} / \mathrm{mL}$. Aldara cream was obtained from 3M (St. Paul, MN). IMQ and TLR7 ligandphospholipid conjugates (TMX-202, molecular weight 920) were prepared by Interquim (Sant Cugat del Valles, Spain) and by CordenPharma (Liestal, Switzerland), respectively, and formulated in a gel by Dr. Franco Pattarino (School of Pharmacy, Novara, Italy). In order to make equivalent molar concentration with Aldara, 5\% IMQ and 19\% TMX-202 were prepared in the gel formulations. For in vitro assays, the unformulated compounds were dissolved in DMSO as a $10 \mathrm{mM}$ stock solution and kept at $-20^{\circ} \mathrm{C}$ until use. Endotoxin levels in all reagents except TMX-202 were measured using the Endosafe ${ }^{\circledR}$ (Charles River Laboratories, Wilmington, MA). Endotoxin levels of the reagents were $<0.05 \mathrm{EU} / \mu \mathrm{mol}$. Because TMX-202 gave a false positive signal by Endosafe ${ }^{\circledR}$, endotoxin contamination in unformulated TMX-202 was ruled out by the finding of similar potencies of this compound in $\mathrm{Tlr} 4^{-/-}$and wild type bone marrow derived cells [7].

\section{In vitro experiments}

The proinflammatory potencies of IMQ and TMX-202 were tested in the murine macrophage cell line RAW 264.7 and in primary bone marrow derived dendritic cells (BMDC), as previously described [7]. The levels of TNF $\alpha, \mathrm{IL}-1 \beta$, and IL-6 were measured by ELISA [7]. In vitro experiments using human whole blood were performed by Bioneer A/S (Copenhagen, Denmark). Briefly, TMX-202 was diluted with medium to prepare 10-time-concentrated stock solution of each test concentration. $20 \mu \mathrm{L}$ of TMX-202 stock solution was added to $180 \mu \mathrm{L}$ blood. The blood cells were cultured for $24 \mathrm{hrs}$ in RPMI-1640 (Invitrogen, Carlsbad, CA), supplemented with $10 \%$ fetal calf serum, penicillin and streptomycin (complete RPMI).

To make human PBMC conditioned media, $10^{6} / \mathrm{mL}$ human peripheral blood mononuclear cells (PBMC) were isolated in a Ficoll gradient and stimulated with TMX-202 for 24 hrs in complete RPMI [7]. Culture supernatants of PBMC without TMX-202 served as a negative control. The stimulated culture supernatants (conditioned media; $\mathrm{CM}$ ) were kept frozen at $-20^{\circ} \mathrm{C}$ until use. The human keratinocyte cell line, $\mathrm{HaCaT}$, was purchased from ATCC (Manassas, VA) and cultured in complete RPMI. Squamous carcinoma cell lines (SCC-13) were obtained from the Harvard Skin Disease Center (Boston, MA) and grown in keratinocyte serum free medium (Invitrogen) with $25 \mu \mathrm{g} / \mathrm{mL}$ of bovine pituitary extract, $0.2 \mathrm{ng} / \mathrm{mL}$ of epidermal growth factor, $0.4 \mathrm{mM}$ $\mathrm{CaCl}_{2}$ and antibiotics. CM was diluted with complete RPMI or keratinocyte media by 2,4 or 8 fold $2 \times 10^{4} \mathrm{HaCaT}$ cells or SCC-13 cells were cultured with diluted condition media for $18 \mathrm{hrs}$ and cell viabilities were quantitated by MTT assay (Invitrogen). In some experiments, $\mathrm{HaCaT}$ cells were incubated with 2 fold diluted media in the presence of antibodies against human interferon gamma (IFN- $\gamma)(10 \mathrm{~g} / \mathrm{mL}$, clone BMS107, eBioscience), TRAIL ( $25 \mu \mathrm{g} / \mathrm{mL}$, clone 550912, $\mathrm{BD}$ Bioscience), or tumor necrosis factor alpha (TNF $\alpha)$ $(25 \mu \mathrm{g} / \mathrm{mL}$, clone htnfa-mab, Invitrogen) for $48 \mathrm{hrs}$. The cell viabilities were determined by MTT assay. IFN- $\gamma$ in the culture supernatants was measured by Luminex beads assay.

\section{Cytochrome P450 enzyme assays and radiolabeled binding study}

The enzyme and binding assays were performed by Ricerca Biosciences LLC (Concord, OH). The percent inhibition of specific enzyme activity or specific binding was determined using radiolabeled specific ligands to the receptors. The detailed methods employed are described on the Ricerca website (https://pharmacology.ricerca.com/ Catalog/Default.aspx). Control samples contained 0.1 or $1 \%$ DMSO. More than $50 \%$ inhibition in drug treated samples was considered significant.

\section{PD/PK study}

Aldara, IMQ gel or TMX-202 gel were applied on the shaved back skin of wild type, $T l r 7^{-/-}$or wild type/Tlr $77^{-/-}$ chimeric mice using $1 \mathrm{~cm}^{2}$-window stencils. Mice were bled at indicated time points. The levels of TNF $\alpha, \mathrm{KC}$, and IL-6 were measured by Luminex assay (Millipore, Billerica, MA). In preliminary experiments, IMQ concentration in serum after Aldara or 5\% IMQ gel were determined as described previously [14]. The samples were analyzed by Agilent 1100 LC/MSD with a Supelco Discovery HS C18 column $(2.1 \times 50 \mathrm{~mm})$, eluted with a linear gradient from $20 \%$ to $90 \%$ methanol in $0.1 \%$ aqueous trifluoroacetic acid at a flow rate of $0.2 \mathrm{~mL} / \mathrm{min}$ monitored by MS/MS and Area Under Curve (AUC) was normalized with an internal standard. To compare the plasma concentrations of TMX202 and IMQ after treatment with TMX-202 gel or Aldara, samples from different time points after the treatment were analyzed by NiKem Research (Baranzate, Milano, Italy). 


\section{Determination of maximum tolerance dose by intravenous administration}

For intravenous administration, IMQ and TMX-202 were formulated in $40 \%$ beta-cyclodextrin in saline $(\mathrm{pH} \mathrm{6)}$ ) and in $3 \%$ dimethyl sulfoxide, $20 \%$ beta-cyclodextrin or $0.1 \%$ lactic acid in saline ( $\mathrm{pH}$ 6), respectively. Wild type, $\mathrm{Tl} \mathrm{r}^{-/-}$ or $\mathrm{Myd} 88^{-/}$mice were intravenously injected with an escalated dose of IMQ $(5,15$, or $30 \mathrm{mg} / \mathrm{kg})$ or TMX-202 (50, 150 or $200 \mathrm{mg} / \mathrm{kg}$ ). The clinical signs and the body weights of the mice were monitored for 8 days.

\section{Induction and treatment of abnormal skin proliferation in the Inv-Myc mouse model}

Inv-MycER mice were shaved on the dorsal skin $24 \mathrm{hrs}$ before tamoxifen treatment. Keratinocyte proliferation was induced by daily application of $5 \mathrm{mg} / \mathrm{mL}$ tamoxifen from days 0 to 7 . Mice were treated with TMX-202 gel on days $0,2,4,6,8$, and 10. Sera samples were collected $2 \mathrm{hrs}$ after the first gel application. On day 11 , mice were sacrificed. The skin samples were fixed in buffered formalin and embedded in paraffin. Ten $\mu \mathrm{m}$ sections were cut and stained with hematoxylin and eosin (H\&E) by the Histology Shared Resources at Moores UCSD Cancer Center. Histological examination was performed in a blinded fashion. The criteria for histopathologic semi-quantitative severity scores used were as follows; for acanthosis, average thickness of the corneal layer was defined as: Score $0=$ one layer of epidermal cells, $1=2-3$ layers, $2=4-5$ layers, $3=6-7$ layers, $4=>7$ layers; mitosis figures were counted over 10 high power fields, $(\times 630)$, and scored as: Score $0=0$ mitosis, $1=1-3$ mitosis, $2=3-4$ mitosis, $3=5-7$ mitosis, $4=>7$ mitosis; and for hyperkeratosis, the severity scores represented the total average thickness of the keratin layer as: Score $0=1-2$ cell layers, $1=3-5$ cell layers, $2=5-7$ cell layers, $3=8-9$ cell layers, $4=>9$ cell layers

\section{Statistical Analysis}

Prism 5 (GraphPad Software, San Diego, CA) software was used for statistical analyses. Data were plotted and fitted by non-linear regression assuming a Gaussian distribution or sigmoidal dose responses with uniform standard deviations between groups. Differences between two groups were assessed by the Student $t$ test. Differences between multiple groups were assessed by two-way ANOVA with Bonferroni's post hoc, or Dunnett's post hoc test. A value of $p<0.05$ was considered statistically significant.

\section{Results}

TMX-202 is highly potent in vitro, but a weak inducer of systemic cytokines in vivo. The potencies of the TLR7 ligand-phospholipid conjugate, TMX-202, and of IMQ were initially compared in vitro using the murine macrophage cell line RAW 264.7 as well as primary mouse bone marrow derived dendritic cells (BMDC). IMQ acts by secretion of inflammatory cytokines, such as TNF $\alpha$,

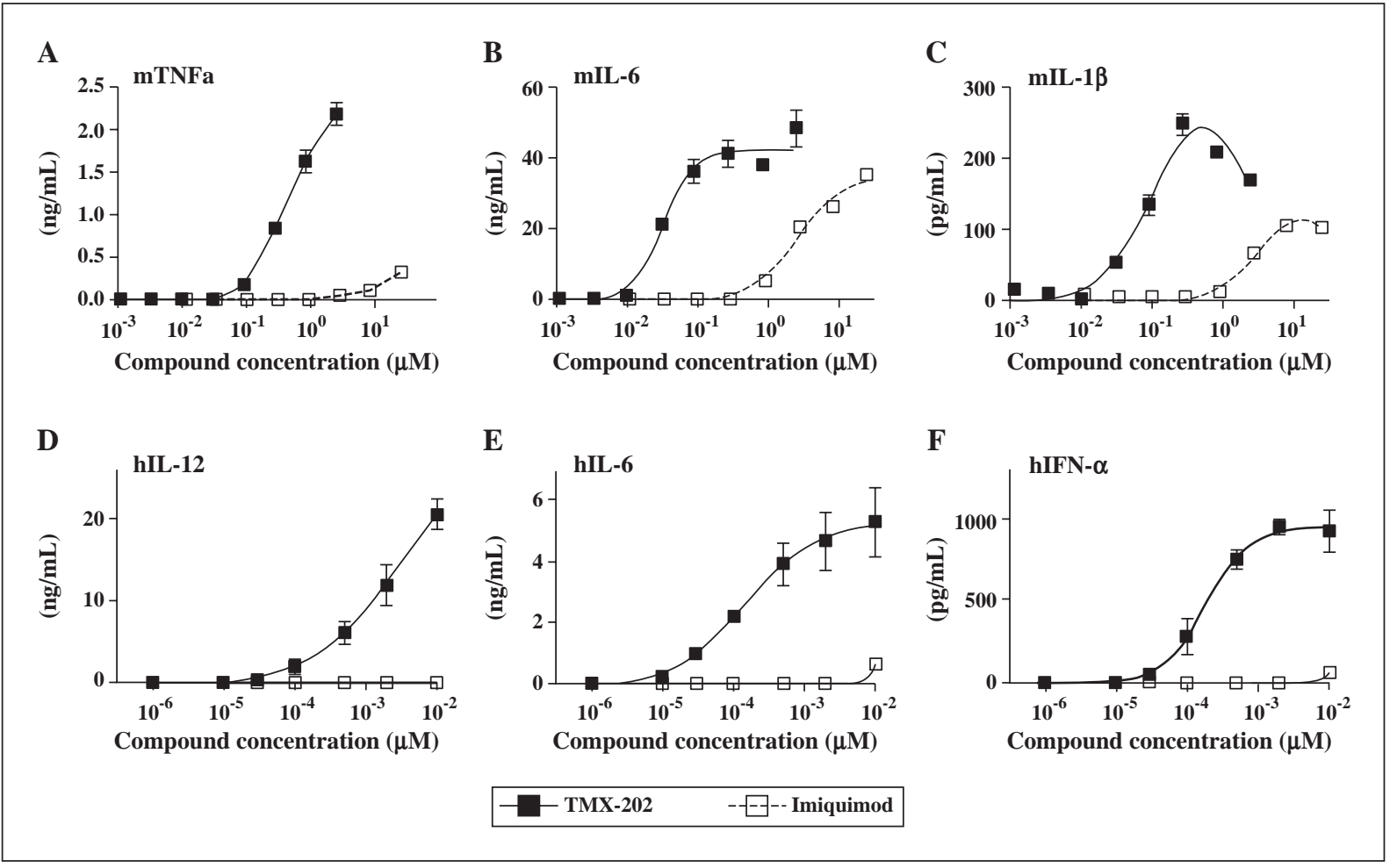

Figure 1. TMX-202 is more potent than IMQ. Murine macrophage RAW 264.7 cells (A), primary BMDC (B and C) or human whole blood (D, E and $\mathbf{F}$ ) were treated with graded doses of IMQ or TMX-202 overnight. The levels of murine (m) TNF $\alpha(\mathbf{A})$, mIL-6 (B), mIL1 $\beta$ (C), human (h)IL-12 (D), hIL-6 (E), and hIFN- $\alpha(\mathbf{F})$ were determined by ELISA. Data are mean \pm SEM and representative of two independent experiments showing similar results $(\mathbf{A}, \mathbf{B}$ and $\mathbf{C})$ and pooled data from three independent donors (D, E and $\mathbf{F})$. 
IL-6, IL-1 and type I IFN, to treat viral infection and nonmelanoma skin cancer $[15,16]$. We, therefore, evaluated murine (m)TNF $\alpha$, mIL- 6 , and mIL- $1 \beta$ secreted by RAW cells and mBMDC, to compare the immunostimulatory potencies of IMQ and TMX-202. TMX-202 was a more potent cytokine inducer in both cell types (figures $1 A-C$ ). The higher potency of TMX-202 was also observed in a human whole blood assay (figures $1 D-F$ ).

To compare the in vivo pharmacodynamics of TMX-202 and IMQ, the two agents in a gel formulation were applied on the skin of mice, and the levels of TNF $\alpha$, IL- 6 or KC in the blood were measured thereafter (figure 2A). TMX-202 gel induced significantly lower levels of these cytokines and this effect was sustained up to $6 \mathrm{~h}$ after application compared to the IMQ gel. In contrast, a high cytokine release by Aldara or IMQ gel was observed one hour after drug application (figure 2A). To evaluate the systemic absorption of IMQ and TMX-202, serum concentrations of the compound were compared (figure $2 B$ ). In the preliminary experiment, IMQ concentrations in sera $1 \mathrm{~h}$ after treatment of Aldara and 5\% IMQ gel showed no significant differences (figure 7), Aldara was used to compare with systemic absorption of TMX-202 in the gel. The serum level of TMX202 one hour after the application was significantly lower than that of IMQ after administration of Aldara (2.6 \pm 0.6 vs $139.4 \pm 61.2 \mathrm{pg} / \mathrm{mL}$, respectively $\mathrm{p}<0.05$ ).

Cytokine induction by TMX-202 depends on TLR7 in radiosensitive cells. To confirm that the induction of proinflammatory cytokines by TMX-202 was TLR7 dependent, we used $T l r 7^{-/}$mice as well as $T l r 7^{-/} /$wild type

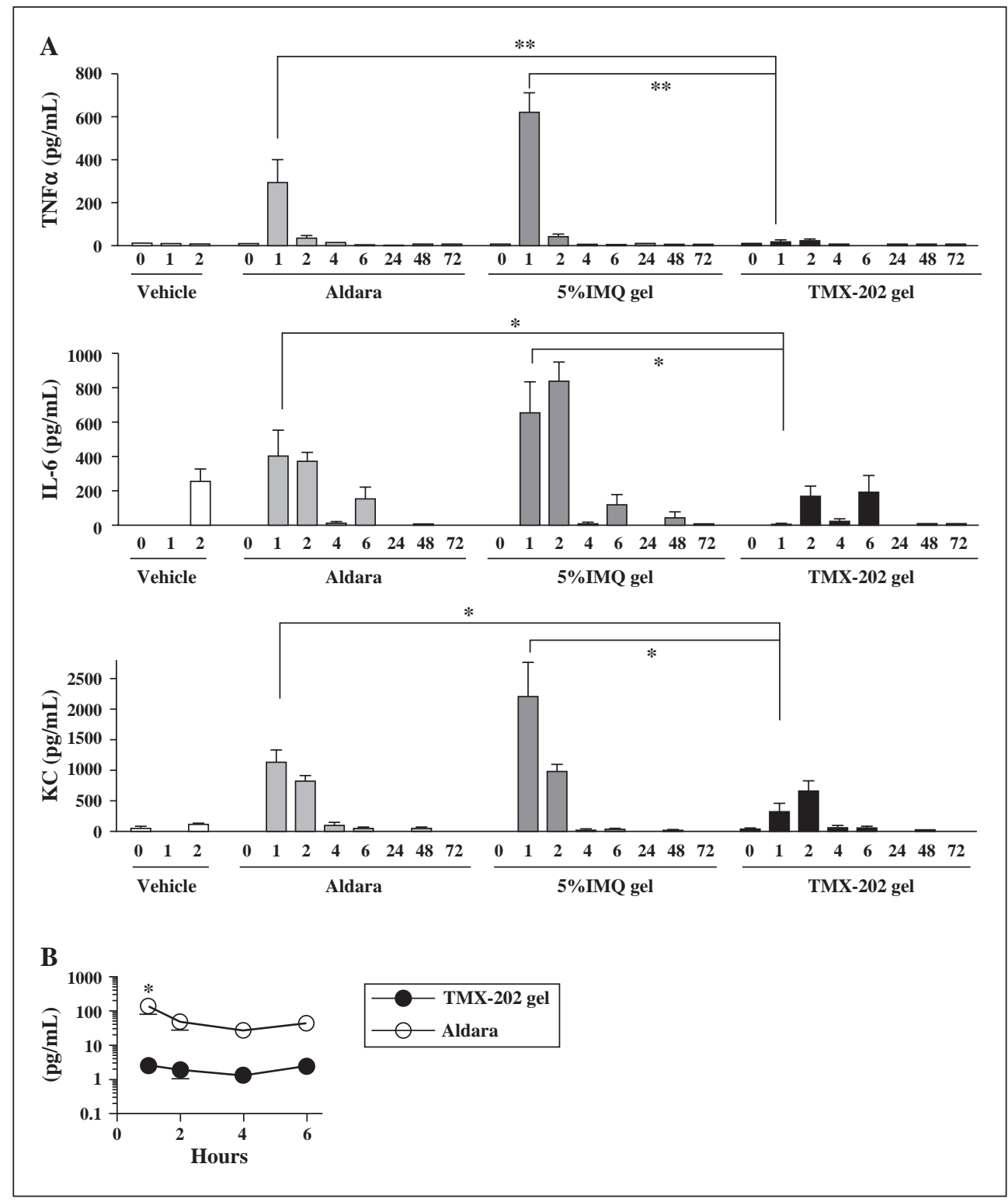

Figure 2. In vivo pharmacodynamics and pharmacokinetics of formulated TMX-202, IMQ or Aldara. (A) Wild type mice were treated with a gel formulation of TMX-202, IMQ, or Aldara using $1 \mathrm{~cm}^{2}$-window stencils. The sera were collected $0,1,2$, $4,6,24,48$, and 72 hrs after application. The levels of TNF $\alpha, \mathrm{KC}$, or IL-6 and were measured by Luminex assay. * and ** denote $\mathrm{p}<0.05$ and $\mathrm{p}<0.001$, respectively, by two-way ANOVA with Bonferroni post hoc testing. (B) The concentrations of TMX-202 and IMQ in the serum $(1,2,4$, and 6 hrs) were determined by Nikem. * denotes $\mathrm{p}<0.05$ by two-way ANOVA with Bonferroni post hoc testing. 

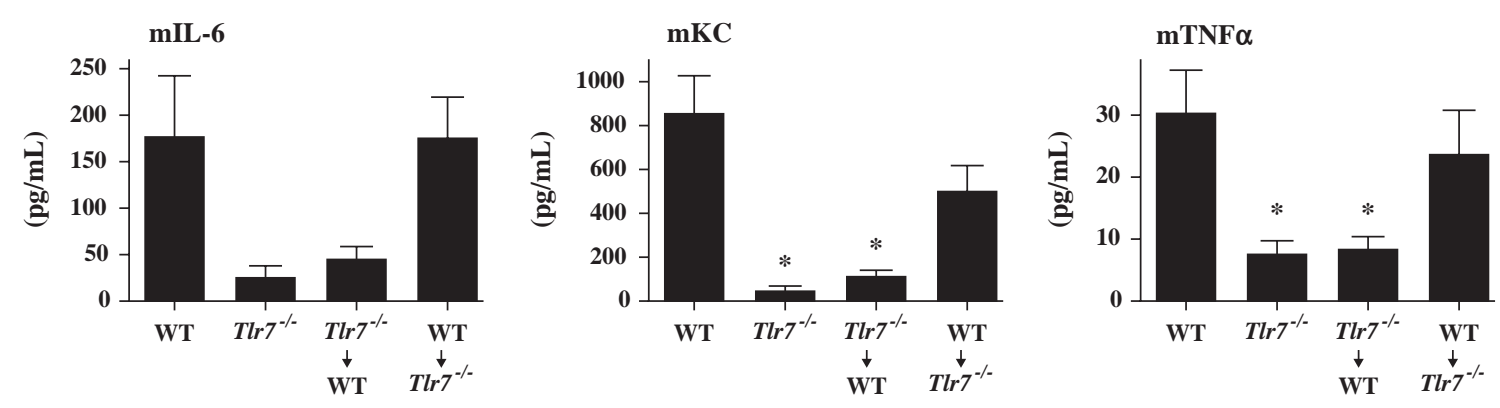

Figure 3. Systemic cytokine induction of TMX-202 depends on radiosensitive cells. WT, $T l r 7^{-/}$, or $T l r 7^{-/} / \mathrm{WT}$ chimeric mice (donor $T l r 7^{-/} \rightarrow$ recipient WT, and donor WT $\rightarrow$ recipient $T l r 7^{--}$) were topically treated with TMX-202 gel and sera samples were collected two hrs after application. Data are mean \pm SEM of pooled data of two independent experiments. $*$ denotes $\mathrm{p}<0.05$ compared to WT mice by one way ANOVA with Dunnet's post hoc testing.

(WT) bone marrow chimeric mice. The cytokine induction by TMX-202 was TLR7 dependent, insofar as $T l r 7^{-/-}$mice were refractory to the TMX-202 gel application (figure 3). TMX-202 maintained its ability to induce proinflammatory cytokines in chimeric $T l r 7^{-/}$mice reconstituted with WT bone marrow, while the induction was diminished in WT mice reconstituted with $T l r 7^{-/}$bone marrow. These data indicate that TLR7 in radiosensitive cells were responsible for most of the cytokine induction by TMX-202.

\section{The maximum tolerated dose (MTD) of TMX-202 is higher than IMQ}

To further compare the toxicity of IMQ and TMX202, the MTD of both compounds were determined in C57BL/6 mice after intravenous administration. The MTD values of IMQ and TMX-202 were $15 \mathrm{mg} / \mathrm{kg}$ (approximately $62.5 \mu \mathrm{moles} / \mathrm{kg}$ ) and $150 \mathrm{mg} / \mathrm{kg}$ (approximately $163 \mu$ moles $/ \mathrm{kg}$ ), respectively. To evaluate acute toxicities, body weights were monitored after a single intravenous injection of the MTD of the two compounds (figure 4). TMX-202 injected mice showed a significant reduction in body weight over two days (figure 4A). These were TLR7 specific effects as no significant body weight loss was observed in either $\mathrm{Tlr}^{-/-}$or $\mathrm{Myd} 88^{-/-}$mice (figure $4 \mathrm{~A}$ ). In contrast, the administration of the MTD of IMQ did not result in body weight changes in WT, $T l r 7^{-/}$or Myd $88^{-/-}$mice (figure $4 B$ ). Potential off-target interactions of TMX-202 with non-specific receptors were evaluated by radiolabeled binding assays performed by Ricerca Biosciences. TMX-202 showed low affinity to receptors for norepinephrine, dopamine and androgen. In contrast, IMQ inhibited the activity of the CYP450 isoenzyme 1A2 and exhibited affinities to adenosine $\mathrm{A} 1$, adenosine $\mathrm{A}_{2 \mathrm{~A}}$, adrenergic $\alpha_{1 \mathrm{~A}}$, adrenergic $\alpha_{1 \mathrm{D}}$, adrenergic $\alpha_{2} \mathrm{~A}$, dopamine $\mathrm{D}_{1}$, histamine $\mathrm{H}_{2}$, imidazoline $\mathrm{I}_{2}$ sodium channel receptors (table 1).

\section{TMX-202 gel reduces acanthosis in inv-MycER mice}

To evaluate the effect of TMX-202 gel on skin malignancy, we employed the inv-MycER mouse model [12]. In this strain, a cDNA encoding human c-myc is fused in frame to the hormone-binding domain of a modified murine estrogen receptor (c-mycERTM), which was cloned downstream of the human involucrin promoter [12, 17]. Reversible activation of c-Myc in skin by tamoxifen (estrogen) application induces benign changes of papillomatosis together with angiogenesis, which resembles hyperplastic actinic keratosis [12]. Induction of the c-Myc gene by local application with tamoxifen results in hyperplasia of suprabasal keratinocytes, with resulting acanthosis (figure 5A). The tamoxifen-conditioned mice were treated topically with TMX-202 gel or Aldara every other day. Macroscopically, the dorsal skins of the TMX-202 treated animals was less reddened and thinner than that of the Aldara-treated mice (figure 5A). Histological examination showed that there was less acanthosis and fewer mitoses in the TMX-202 geltreated skin (figure 5B). The Aldara-treated mice showed slight hyperkeratosis (figure 5B). The TMX-202 gel did not cause significant systemic cytokine induction while the Aldara cream did, consistent with the data in wild type mice (figure 5C).

\section{IFN- $\gamma$ is involved in anti-proliferative effects of TMX-202 on keratinocytes}

In human keratinocytes, a limited panel of TLRs are expressed and TLR7 has been reported as absent or detectable at very low levels [18]. We therefore hypothesized that the anti-proliferative effects of TMX-202 might be mediated by factors released by TLR7 stimulated immune cells. To test this hypothesis, human PBMC were stimulated with TMX-202 and the culture supernatants were used as conditioned media (TMX-202+CM). The average IFN- $\gamma$ levels in the TMX-202+CM from three donors was $204 \pm 113 \mathrm{pg} / \mathrm{mL}$. HaCaT or SCC-13 cells were treated with TMX-202+CM, or TMX-202 alone for $48 \mathrm{hrs}$ and the cell viabilities were compared. In both cell lines, TMX-202+CM significantly reduced the cell viabilities (figure 6A). As expected, TMX-202 alone did not alter the cell viability (figure $6 A$ ). To further identify the mediators of the anti-proliferative action, neutralizing antibodies against IFN- $\gamma$, TRAIL or TNF $\alpha$ were added to TMX$202+$ CM. Only neutralization of IFN- $\gamma$ could significantly reverse the depressed viability of HaCaT cells (figure $6 B$ ), indicating that IFN- $\gamma$ mediated the anti-proliferative effects 

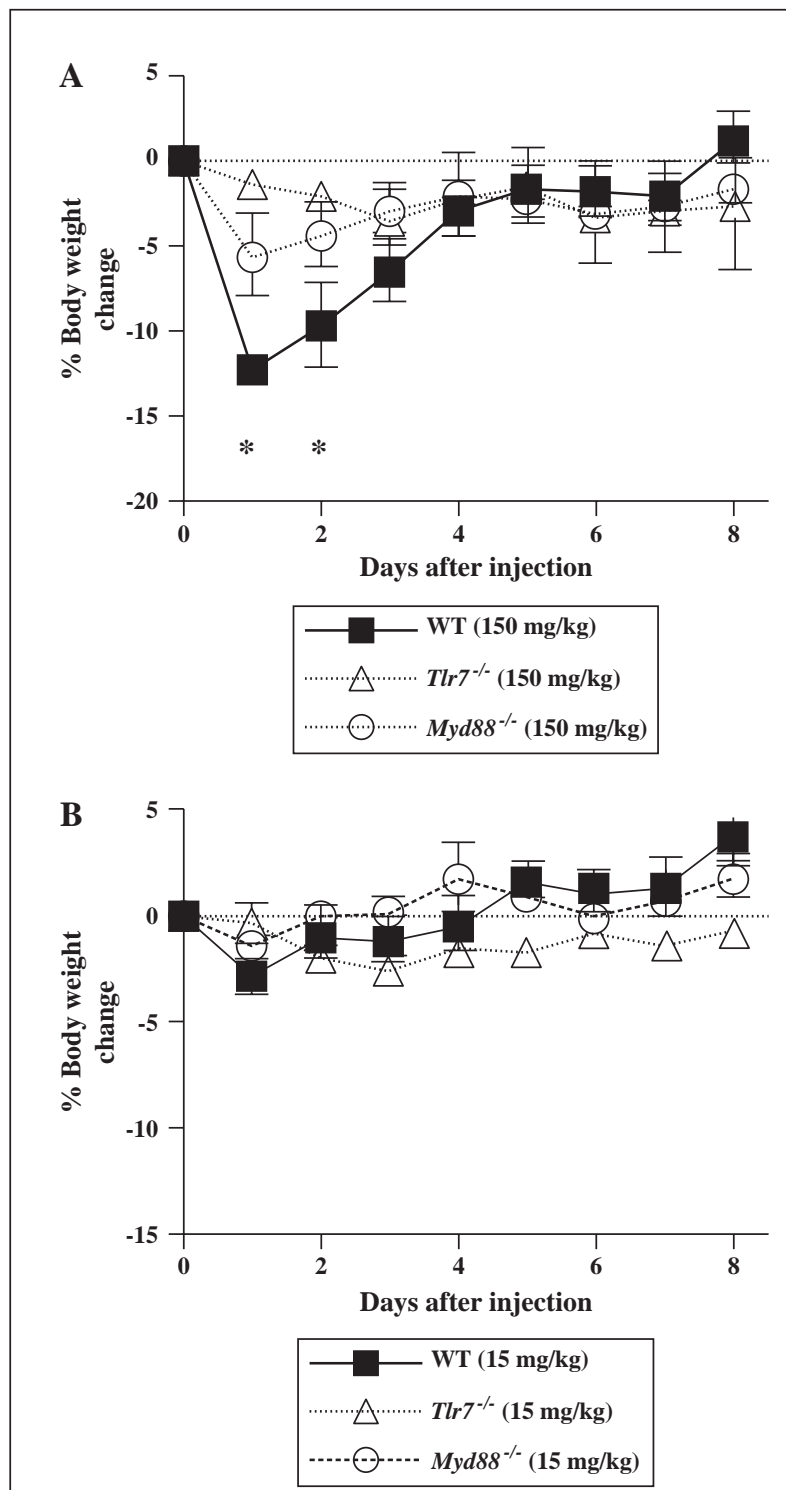

Figure 4. Body weight changes after administration of maximum tolerated dose of IMQ or TMX-202. WT, $M y d 88^{-/}$, and $T l r 7^{-/}$mice were i.v. administered with 150 $\mathrm{mg} / \mathrm{kg}$ TMX-202 (A) and $15 \mathrm{mg} / \mathrm{kg}$ IMQ (B) in $200 \mu \mathrm{L}$. Mice were monitored daily and their body weights were measured. Data are mean \pm SEM of pooled data of two independent experiments. * denotes $\mathrm{p}<0.05$ compared to $M y d 88^{-/-}$, and $T l r 7^{-/}$ mice by two-way ANOVA with Bonferroni post hoc testing.

of TMX-202. To further confirm that TMX-202 induced IFN- $\gamma$ in various donors, PBMC from five independent donors were stimulated with TMX-202. Human PBMC treated with TMX-202 secreted IFN- $\gamma$ significantly higher than vehicle treated controls (figure 6C).

\section{Discussion}

A 5\% cream formulation of IMQ, Aldara is an effective treatment for basal cell carcinoma (BCC), genital warts and actinic keratosis (AK). The predominant mechanism of action of IMQ is induction of cytokines, dependent upon activation of TLR7/8, and induction of Th1 dominant antitumor immune responses [1]. Because of adverse effects at local application sites, the current Aldara treatment regime requires administration three times per week with no treatment intervals $[9,10]$. Our laboratory synthesized the purine analog TLR7 ligand, 9-benzyl- 8-hydroxy2-(2-methoxyethoxy) adenine [3] conjugated to a C-12 phospholipid designated as TMX-202. Although the in vitro immunostimulatory potency of TMX-202 was higher than that of IMQ, topical skin application of TMX-202 gel in vivo did not induce high blood levels of pro-inflammatory cytokines. A PK study showed that TMX-202 was less absorbed into the systemic circulation than IMQ in Aldara cream. In the inv-MycER mouse model, application of TMX-202 gel achieved anti-proliferative effects similar to Aldara with less local inflammation.

An advantage of local treatment is to be able to increase local exposure of drugs without causing systemic adverse effects. Therefore, the local administration of immune-stimulatory compounds ideally induces inflammatory cytokines in the limited target area and avoids systemic cytokine release to reduce adverse effects. Although the in vitro potency of TMX-202 gel was 100 times higher than IMQ, the skin application of the drug did not cause the spiked increase in systemic cytokine release that was observed with Aldara. The minimal systemic cytokine induction by TMX-202 gel was also seen in tamoxifentreated Inv-MycERTAM mice, which might have exhibited compromised epidermal permeability. Collectively, our data suggest that the lower cytokine induction is due to the low systemic absorption of TMX-202. The skin surface is covered by extremely hydrophobic epidermal lipids forming highly structured layers. The lipophilic nature of TMX-202 may enable local penetration and residence within the skin layers, thus maintaining high local concentration, while systemic absorption of the drug is minimized.

In experiments comparing maximum tolerated doses (MTD) of IMQ and TMX-202 in mice, the latter compound was better tolerated, with an MTD of $150 \mathrm{mg} / \mathrm{kg}$ (approximately $163 \mu \mathrm{moles} / \mathrm{kg}$ ) compared to an MTD of $15 \mathrm{mg} / \mathrm{kg}$ (approximately $62.5 \mu \mathrm{moles} / \mathrm{kg}$ ) for IMQ. Moreover, experiments performed with wild type, $\mathrm{Tlr} 7^{-/-}$and $\mathrm{Myd} 88^{-/-}$mice showed that the toxic effects of TMX-202 were TLR7- and MyD88-dependent, while IMQ exerted adverse effects that were TLR7 and MyD88 independent. Thus, the adverse effects of IMQ are due at least in part to undefined off-target interactions, whereas the observed side effects of TMX202 at higher doses are fully dependent on the presence of TLR7 and the respective MyD88 signaling pathway. Since TMX-202 is 100 times more potent than IMQ in causing TLR7-dependent cytokine induction, we conclude that the therapeutic window of TMX-202 is significantly greater than that of IMQ.

The systemic cytokine induction by TMX-202 required TLR7 expression in radiosensitive cells, as indicated by studies using bone marrow chimeric mice. In the MTD study, the anorexia and body weight loss observed in the mice receiving the maximum tolerated dose of TMX-202 was similar to the anorexic behavior caused by TNF $\alpha$, consistent with the TLR7 specificity of the drug [19]. In contrast, when mice were given IMQ at the MTD (which was a 10-fold lower dose than TMX-202), the animals did 
Table 1. Percent inhibition ${ }^{* 1}$ of specific radioligand binding or enzyme activity by IMQ and TMX-202

\begin{tabular}{|c|c|c|c|c|c|}
\hline Target Compounds & Species & $\begin{array}{c}\text { Incubation } \\
\text { temperature }\left({ }^{\circ} \mathbf{C}\right)\end{array}$ & $\begin{array}{l}\text { Incubation } \\
\text { time (min) }\end{array}$ & Imiquimod ${ }^{* 2}$ & TMX-202*2 \\
\hline CYP450, $1 \mathrm{~A} 2 * 3$ & human & 37 & 30 & $66^{* 4}$ & -4 \\
\hline CYP450, 2C19*3 & human & 37 & 45 & 10 & 1 \\
\hline CYP450, 2C9*3 & human & 37 & 45 & 3 & -4 \\
\hline CYP450, 2D6*3 & human & 37 & 45 & 29 & 0 \\
\hline CYP450, 3A4*3 & human & 37 & 30 & 1 & -1 \\
\hline Adenosine $\mathrm{A}_{1}$ & human & 25 & 90 & 50 & 10 \\
\hline Adenosine $\mathrm{A}_{2 \mathrm{~A}}$ & human & 25 & 90 & 89 & -8 \\
\hline Adenosine $\mathrm{A}_{3}$ & human & 25 & 60 & 16 & 6 \\
\hline Adrenergic $\mathrm{a}_{1 \mathrm{~A}}$ & rat & 25 & 60 & 65 & -11 \\
\hline Adrenergic $\mathrm{a}_{1 \mathrm{~B}}$ & rat & 25 & 60 & 40 & 0 \\
\hline Adrenergic $\mathrm{a}_{1 \mathrm{D}}$ & human & 25 & 60 & 76 & 6 \\
\hline Adrenergic $\mathrm{a}_{2 \mathrm{~A}}$ & human & 25 & 60 & 58 & 3 \\
\hline Adrenergic $\beta_{1}$ & human & 25 & 120 & 19 & 18 \\
\hline Adrenergic $\beta 2$ & human & 25 & 60 & 18 & 3 \\
\hline Androgen (Testosterone) AR & rat & 4 & 240 & 7 & 55 \\
\hline Bradykinin B1 & human & 25 & 60 & 4 & 3 \\
\hline Bradykinin B2 & human & 25 & 60 & -1 & -2 \\
\hline Calcium Channel L-Type, Benzothiazepine & rat & 4 & 180 & 4 & 1 \\
\hline Calcium Channel L-Type, Dihydropyridine & rat & 25 & 90 & -14 & 10 \\
\hline Calcium Channel N-Type & rat & 4 & 30 & 2 & -12 \\
\hline Cannabinoid CB1 & human & 37 & 90 & -4 & -14 \\
\hline Dopamine D1 & human & 37 & 120 & 66 & 15 \\
\hline Dopamine D24 & human & 37 & 120 & 14 & 25 \\
\hline Dopamine D3 & human & 37 & 120 & 18 & 15 \\
\hline Dopamine D4.2 & human & 25 & 120 & 2 & 14 \\
\hline Endothelin $\mathrm{ET}_{\mathrm{A}}$ & human & 37 & 120 & 8 & 13 \\
\hline Endothelin $\mathrm{ET}_{\mathrm{B}}$ & human & 25 & 120 & 0 & -7 \\
\hline Epidermal Growth Factor (EG F) & human & 25 & 60 & -1 & 6 \\
\hline Estrogen ERa & human & 25 & 120 & 0 & 7 \\
\hline $\mathrm{GABA}_{\mathrm{A}}$, Flunitrazepam, Cent ral & rat & 25 & 120 & 37 & -23 \\
\hline GABAA, Muscimol, Central & rat & 4 & 10 & 19 & -3 \\
\hline GABAB I A & human & 25 & 180 & -26 & -20 \\
\hline Glucocorticoid & human & 25 & 120 & -2 & -1 \\
\hline Glutamate, Kainate & rat & 4 & 60 & -8 & 1 \\
\hline Glutamate, NMDA, Agonism & rat & 4 & 20 & 2 & 10 \\
\hline Glutamate, NMDA, Glycine & rat & 4 & 30 & -6 & 20 \\
\hline Glutamate, NMDA, Phencyclidine & rat & 25 & 45 & 4 & 2 \\
\hline Histamine $\mathrm{H}_{1}$ & human & 25 & 180 & 8 & -6 \\
\hline Histamine $\mathrm{H}_{2}$ & human & 25 & 120 & 63 & 40 \\
\hline Histamine $\mathrm{H}_{3}$ & human & 25 & 120 & 4 & -14 \\
\hline Imidazoline $\mathrm{I}_{2}$, Central & rat & 25 & 30 & 57 & -3 \\
\hline Interleukin IL-1 & mouse & 37 & 120 & 5 & 5 \\
\hline Leukotriene, Cysteinyl CysLT 1 & human & 25 & 30 & 10 & 10 \\
\hline Melatonin $\mathrm{MT}_{1}$ & human & 25 & 180 & 32 & 3 \\
\hline Muscarinic $\mathrm{M}_{1}$ & human & 25 & 120 & 16 & 0 \\
\hline Muscarinic $\mathrm{M}_{2}$ & human & 25 & 120 & 3 & 0 \\
\hline Muscarinic $\mathrm{M}_{3}$ & human & 25 & 120 & 6 & -6 \\
\hline Neuropeptide Y Y 1 & human & 37 & 60 & 13 & 11 \\
\hline Neuropeptide $\mathrm{Y} \mathrm{Y}_{2}$ & human & 37 & 120 & 6 & 4 \\
\hline
\end{tabular}


Table 1. (Continued)

\begin{tabular}{|c|c|c|c|c|c|}
\hline Target Compounds & Species & $\begin{array}{c}\text { Incubation } \\
\text { temperature }\left({ }^{\circ} \mathbf{C}\right)\end{array}$ & $\begin{array}{l}\text { Incubation } \\
\text { time (min) }\end{array}$ & Imiquimod $* 2$ & TMX-202*2 \\
\hline Nicotinic Acetylcholine & human & 25 & 60 & 3 & 9 \\
\hline Nicotinic Acetylcholine a1, Bungarotoxin & human & 25 & 120 & 7 & -3 \\
\hline Opiate $51(\mathrm{OP} 1, \mathrm{DOP})$ & human & 25 & 60 & 5 & 5 \\
\hline Opiate K (OP2, KOP) & human & 25 & 60 & 8 & -9 \\
\hline Opiate n (OP3, MOP) & human & 25 & 60 & 14 & 42 \\
\hline Phorbol Ester & mouse & 25 & 60 & 21 & -8 \\
\hline Platelet Activating Factor (PA F) & human & 25 & 180 & 2 & 33 \\
\hline Potassium Channel [ $\left.\mathrm{K}_{\mathrm{ATP}}\right]$ & human & 25 & 120 & -5 & 8 \\
\hline Potassium Channel hERG & human & 25 & 60 & 45 & -4 \\
\hline Prostanoid $\mathrm{EP}_{4}$ & human & 25 & 120 & 6 & 7 \\
\hline Purinergic $\mathrm{P}_{2 \mathrm{x}}$ & rabbit & 25 & 30 & -7 & 9 \\
\hline Purinergic $\mathrm{P}_{2}$, & rat & 25 & 60 & 10 & 8 \\
\hline Rolipram & rat & 4 & 60 & 41 & 21 \\
\hline Serotonin (5-Hydroxytryptam ine) 5 -HT $1 \mathrm{~A}$ & human & 25 & 60 & 19 & -7 \\
\hline Serotonin (5-Hydroxytryptam ine) 5-HT2B & human & 37 & 60 & 45 & 1 \\
\hline Serotonin (5-Hydroxytryptam ine) 5 -HT3 & human & 37 & 60 & 8 & 3 \\
\hline Sigma al & human & 37 & 240 & 15 & -5 \\
\hline Sodium Channel, Site 2289593 & rat & 37 & 60 & 72 & 15 \\
\hline Tachykinin $\mathrm{NK}_{1}$ & human & 4 & 90 & -8 & 23 \\
\hline Thyroid Hormone & rat & 4 & $18 \mathrm{hrs}$ & -5 & 11 \\
\hline Transporter, Dopamine (DAT) & human & 4 & 180 & 25 & 64 \\
\hline Transporter, GABA & rat & 25 & 20 & 2 & 1 \\
\hline Transporter, Norepinephrine (NET) & human & 4 & 180 & 30 & 57 \\
\hline Transporter, Serotonin (5-Hyd roxytryptamine) (SERT) & human & 25 & 60 & 0 & 18 \\
\hline
\end{tabular}

* 1; Assays were performed in duplicates. The values are average of the duplicate data.

*2; $10 \mu M$

*3; Activities of cytochrome (CYP 450) isozyme were determined by spectrofluorimetric quantitation of 3-cyano-7-hydroxycoumarin. Other target compounds were studied using the radioligand assays.

*4:Bold letter indicates $\geq 50 \%$ inhibition.

not show any body weight loss. Thus, it is likely that this phenotype was not observed with IMQ because of its lower $\mathrm{TNF} \alpha$ inducing activity.

As part of the toxicity assessments, inhibition of CYP450 isozymes by TMX-202 and IMQ were compared. While IMQ possibly bound to multiple adenosine or adrenergic receptors and inhibited CYP450 enzyme activity, TMX202 lacked these effects. CYP450 inhibition can alter drug metabolism. Adenosine receptor ligands can affect cardiac rhythm. Collectively, these results indicate that TMX-202 gel should have fewer TLR7-independent off-target effects, compared to IMQ.

Actinic keratosis (AK) is a common dysplastic cutaneous lesion, which can progress to squamous cell carcinoma [20]. In human $\mathrm{AK}$ and squamous cell carcinoma, Myc gene aberrations are often observed [20]. In inv-MycERTAM transgenic mice, an acutely regulable form of Myc is targeted to the epidermis via the involucrin promoter and is locally activated by topical application of estrogen (tamoxifen). Activation of the cMyc gene in the suprabasal keratinocytes of these mice induces pre-malignant lesions similar to human hyperproliferative and dysplastic states [12]. Reversible activation of c-Myc in skin induces a complex neoplastic phenotype by a single oncogenic lesion state. In this model, sustained activation of c-Myc is suffi- cient to induce papillomatosis together with angiogenesis, resembling hyperplastic AK, a commonly observed human precancerous epithelial lesion [12]. It should be noted that the pre-malignant changes regress after the deactivation of $\mathrm{cMyc}$ and no malignancy transformation is observed in this model. Systemic absorption of IMQ after treatment with Aldara and 5\% IMQ gel was similar (figure 7). Also, Walre et al. [21] have published that the formulation material of Aldara causes inflammasome-related immune reactions. Since the formulation influences the efficacy, the efficacy of TMX-202 gel was compared to Aldara; IMQ in the specific dosage formulation with the active ingredients. Application of a TMX-202 gel to the dysplastic skin lesion did not cause the severe redness that was seen in Aldara-treated mice, suggesting fewer local inflammatory responses (figure 4A). The data thus indicated that TMX-202 gel is as efficacious as Aldara without causing severe local inflammatory effects.

The expression level of TLR7 in keratinocytes is low or absent $[18,22,23]$. Our study indicated that a soluble factor secreted by TMX-202-treated immune cells attenuated the viability of HaCaT keratinocytes. Neutralization of IFN$\gamma$ significantly diminished the growth inhibitory effects of TMX-202 conditioned media toward $\mathrm{HaCaT}$ keratinocytes. A previous report by Gorski indicated that IMQ does not 


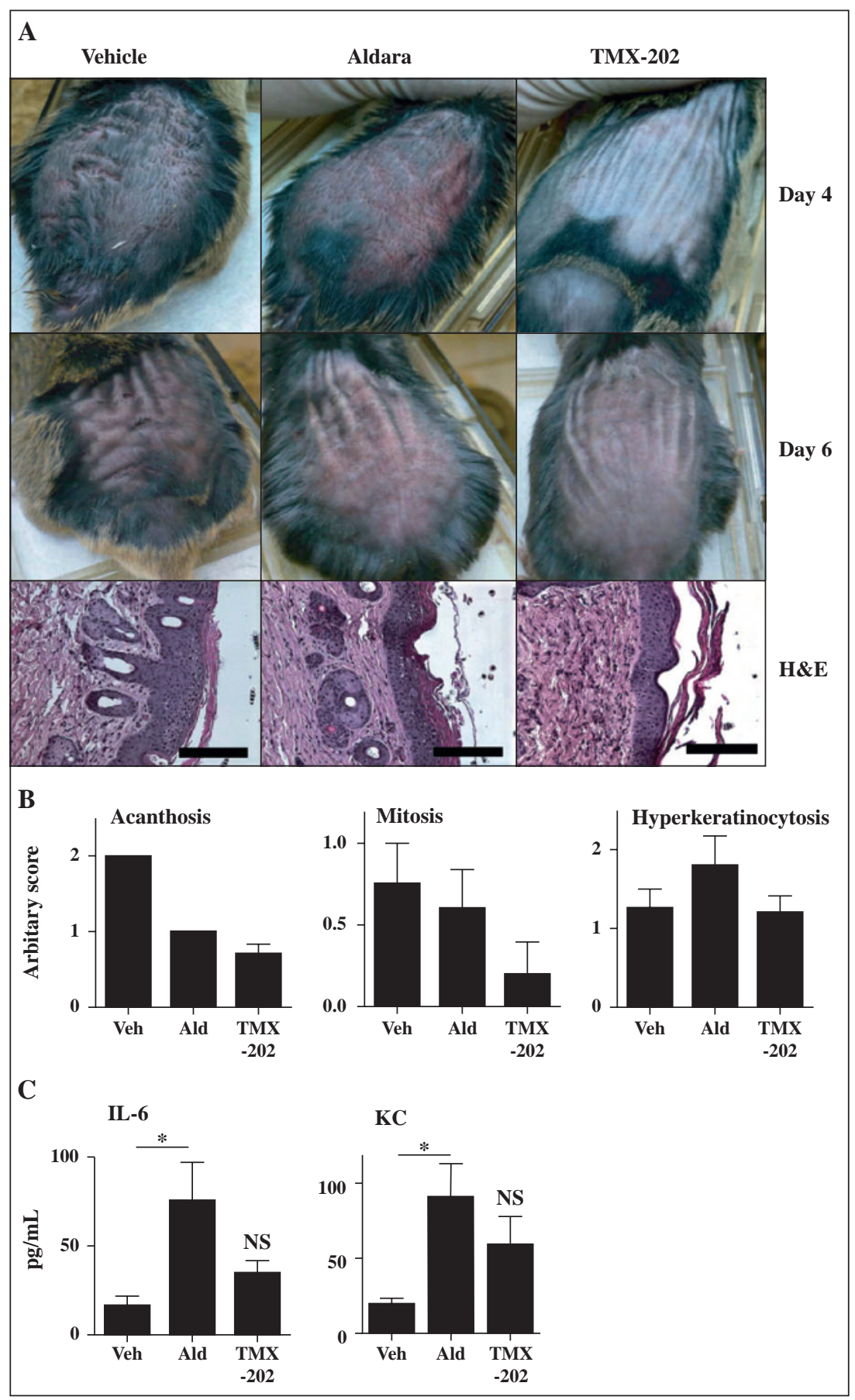

Figure 5. In vivo efficacy evaluation of TMX-202 gel. The back skin of Inv-Myc mice $(\mathrm{n}=5)$ was treated with $5 \mathrm{mg} / \mathrm{mL}$ tamoxifen daily for days 0 to 7. Mice were treated with Aldara, TMX-202 or vehicle gel on days 0, 2, 4, 6, 8, and 10. (A and B) On days 4 and 6, the gross appearance of the skin was recorded (A). On day 11, the skin samples were collected and hematoxylin/eosin-stained samples were evaluated and scored for acanthosis, mitosis, or hyperkeratinocytosis (B). Data shown are representative of two independent experiments. (C) Levels of IL-6, and KC in sera samples 2 hrs after the first application. Data shown are mean \pm SEM of pooled data of two independent experiments. $*$ denotes $\mathrm{p}<0.05$ by one way ANOVA with Bonferroni post hoc test.

stimulate IFN- $\gamma$ secretion in human PBMC up to $1 \mu \mathrm{M}$ [24]. In our study, TMX-202 induced significantly higher IFN- $\gamma$ compared to vehicle control. The higher pro-inflammatory potency of TMX-202 compared to IMQ demonstrated by our in vitro experiment (figure 1) might be due to ability to induce IFN- $\gamma$ by PBMC. In addition, trans-dermally administered TMX-202 might be locally sustained, contributing to an increased local concentration of TMX-202. Although not studied, IFN- $\alpha$ may also contribute, since TLR7 agonists induce high concentrations of type I IFN [7], and the 


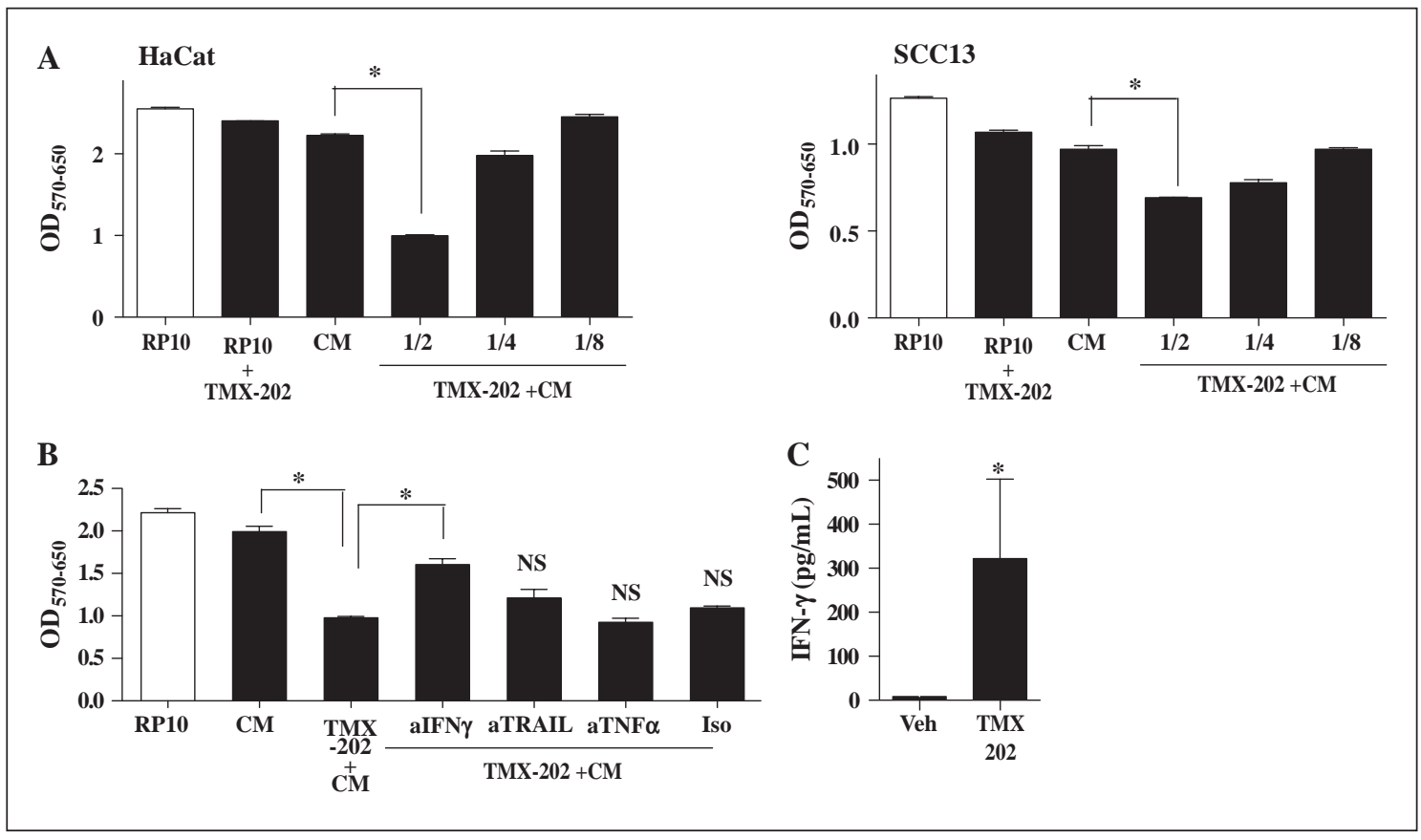

Figure 6. TMX-202 conditioned media suppressed the growth of keratinocytes. Human PBMC $\left(10^{6} / \mathrm{mL}\right)$ were incubated with $1 \mu$ M TMX-202 in complete RPMI and the culture supernatants were used as conditioned media (TMX-202+CM). CM without TMX-202 or complete RP-10 were used as controls. (A) HaCaT or SCC13 cells were incubated with TMX-202 +CM at various dilutions $(1 / 2,1 / 4$, or $1 / 8)$ for $48 \mathrm{hrs}$ and the viability of cells was determined by MTT assay. (B) HaCaT cells were treated with 2 fold diluted media in the presence of antibodies against human IFN- $\gamma$, TRAIL or TNF $\alpha$ for 48 hrs. * denotes $\mathrm{p}<0.05$ compared to the CM or TMX-202+ CM by one-way ANOVA with Bonferroni post hoc test. NS denotes "not significant" compared to TMX-202+CM. (C) PBMC from five independent donors were cultured with TMX-202, and IFN- $\gamma$ in the culture supernatant was measured by Luminex beads assay. ${ }^{*}$ denotes $\mathrm{p}<0.05$ compared to vehicle treatment by Student $t$ test.

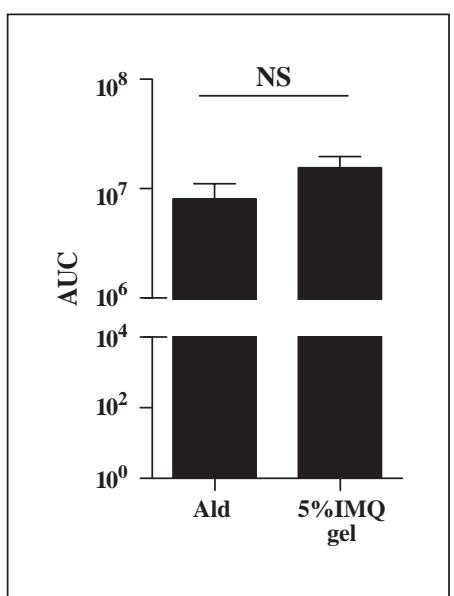

Figure 7. Serum levels of IMQ are similar after treatment with Aldara and 5\% IMQ gel. C57BL/6 were applied with Aldara $(n=4)$ or $5 \%$ IMQ gel $(n=5)$ and sera were collected $1 \mathrm{~h}$ after treatment. The drug concentrations were determined using IMQ as a internal standard and are expressed as area under the curve (AUC). NS; not significant using Student $t$ test.

combination of IFN- $\alpha$ plus IFN- $\gamma$ induces keratinocyte cell death, mediated by the TRAIL receptor [25]. Several cell types present in the skin, including natural killer cells and mast cells are known to synthesize IFN- $\gamma$, [26, 27]. Further studies are necessary to identify the responsible cell type(s) producing IFN- $\gamma$.

Aldara has been widely used and demonstrated to be safe and effective. However, because of local inflammatory reactions, an intermittent dosage regimen is required. Recently, lower IMQ concentrations $(2.5 \%$ and $3.75 \%)$ in new formulations have been tested for daily application, without a no-treatment interval, to shorten the treatment period [28]. The gel formulation of TMX-202, is minimally proinflammatory and thus could be applied daily. The drug's efficacy in the inv-MycER mouse model was comparable to Aldara, without causing local skin reactions. The systemic absorption of TMX-202 was significantly less than IMQ in Aldara, with insignificant off target effects. Thus, the results indicate that TMX-202 should be an efficacious, alternative to Aldara in skin proliferative diseases with fewer adverse effects.

Disclosure. Acknowledgements. We thank Drs. Stella Pelengaris and Michael Kahn, University of Warwick, UK, for providing the Myc transgenic mice. Financial support: This work was supported by Telormedix SA. Conflicts of interest: $A B, R M, E M, N P$, and JH are former or current employees of Telormedix and did not influence planning experiments and data interpretation. DAC and RO are consultants to Telormedix. Other authors do not have any conflict of interest. 


\section{References}

1. Schon MP, Schon M. TLR7 and TLR8 as targets in cancer therapy. Oncogene 2008; 27: 190-9.

2. Gaspari A, Tyring SK, Rosen T. Beyond a decade of $5 \%$ imiquimod topical therapy. J Drugs Dermatol 2009; 8: 467-74.

3. Lee J, Wu CC, Lee KJ, et al. Activation of anti-hepatitis $C$ virus responses via Toll-like receptor 7. Proc Natl Acad Sci U S A 2006; 103: 1828-33.

4. Fukui R, Miyake K. Controlling systems of nucleic acid sensing-TLRs restrict homeostatic inflammation. Exp Cell Res 2012.

5. Hart OM, Athie-Morales V, O'Connor GM, Gardiner CM. TLR7/8-mediated activation of human NK cells results in accessory cell-dependent IFN-gamma production. J Immunol 2005; 175: 1636-42.

6. Kurimoto A, Ogino T, Ichii S, et al. Synthesis and evaluation of 2-substituted 8-hydroxyadenines as potent interferon inducers with improved oral bioavailabilities. Bioorg Med Chem 2004; 12: 1091-9.

7. Chan M, Hayashi T, Kuy CS, et al. Synthesis and immunological characterization of toll-like receptor 7 agonistic conjugates. Bioconjug Chem 2009; 20: $1194-200$.

8. Hayashi T, Chan M, Norton JT, et al. Additive melanoma suppression with intralesional phospholipid-conjugated TLR7 agonists and systemic IL-2. Melanoma Res 2010.

9. Stockfleth E, Meyer T, Benninghoff B, et al. A randomized, double-blind, vehicle-controlled study to assess $5 \%$ imiquimod cream for the treatment of multiple actinic keratoses. Arch Dermatol 2002; 138: 1498-502.

10. Gebaver K, Shumack S, Cowen PS. Effect of dosing frequency on the safety and efficacy of imiquimod $5 \%$ cream for treatment of actinic keratosis on the forearms and hands: a phase II, randomized placebo-controlled trial. Br J Dermatol 2009; 161:897-903.

11. Mosher JS, Lio P. Cytokine dermatitis and febrile seizure from imiquimod. Pediatrics 2012; 129: e519-22.

12. Pelengaris $S$, Littlewood $T$, Khan $M$, Elia G, Evan G. Reversible activation of c-Myc in skin: induction of a complex neoplastic phenotype by a single oncogenic lesion. Molecular cell 1999; 3: 565-77.

13. Hayashi T, Gray CS, Chan $M$, et al. Prevention of autoimmune disease by induction of tolerance to Toll-like receptor 7. Proc Natl Acad Sci U S A 2009; 106: 2764-9.

14. Chan M, Hayashi T, Mathewson RD, et al. Synthesis and Characterization of PEGylated Toll Like Receptor 7 Ligands. Bioconjug Chem $2011 ; 22: 445-54$.
15. Hengge UR, Ruzicka T. Topical immunomodulation in dermatology: potential of toll-like receptor agonists. Dermatol Surg 2004; 30: 1101-12.

16. Stanley MA. Imiquimod and the imidazoquinolones: mechanism of action and therapeutic potential. Clin Exp Dermatol 2002; 27: 5717.

17. Carroll JM, Albers KM, Garlick JA, Harrington R, Taichman LB. Tissue- and stratum-specific expression of the human involucrin promoter in transgenic mice. Proc Natl Acad Sci U S A 1993;90: 10270-4.

18. Mempel $M$, Voelcker $V$, Kollisch $G$, et al. Toll-like receptor expression in human keratinocytes: nuclear factor kappaB controlled gene activation by Staphylococcus aureus is toll-like receptor 2 but not tolllike receptor 4 or platelet activating factor receptor dependent. J Invest Dermatol 2003; 121: 1389-96.

19. Hayashi T, Cottam HB, Chan M, et al. Mast cell-dependent anorexia and hypothermia induced by mucosal activation of Toll-like receptor 7. Am J Physiol Regul Integr Comp Physiol 2008; 295: R12332.

20. Toll $A$, Salgado R, Yebenes $M$, et al. MYC gene numerical aberrations in actinic keratosis and cutaneous squamous cell carcinoma. Brit J Dermatol 2009; 161: 11112-8.

21. Walter $A$, Schafer $M$, Cecconi $V$, et al. Aldara activates TLR7independent immune defence. Nature Com 2013; 4: 1560.

22. Terhorst $D$, Kalali $B N$, Ollert $M$, Ring J, Mempel $M$. The role of toll-like receptors in host defenses and their relevance to dermatologic diseases. Am J Clin Dermatol 2010; 11:1-10.

23. Miller LS, Modlin RL. Toll-like receptors in the skin. Sem Immunopathol 2007; 29: 15-26.

24. Gorski KS, Waller EL, Biornton-Severson J, et al. Distinct indirect pathways govern human NK-cell activation by TLR-7 and TLR-8 agonists. Int Immunol 2006; 18: 111 15-26.

25. Chaturvedi V, Bodner B, Qin JZ, Nickoloff BJ. Knock down of p53 levels in human keratinocytes increases susceptibility to type I and type II interferon-induced apoptosis mediated by a TRAlL dependent pathway. J Dermatol Sci 2006; 41:31-41.

26. Simon $D$, Kozlowski $E$, Simon $H$. Natural killer $T$ cells expressing IFN-gamma and IL-4 in lesional skin of atopic eczema. Allergy 2009; 64: 1681-4.

27. von Bubnoff $D$, Andres $E$, Hentges F, Bieber T, Michel T, Zimmer J. Natural killer cells in atopic and autoimmune diseases of the skin. $J$ Allergy Clin Immunol 2010; 125: 60-8.

28. Hanke CW, Beer KR, Stockfleth E, Wu J, Rosen T, Levy S. Imiquimod $2.5 \%$ and $3.75 \%$ for the treatment of actinic keratoses: results of two placebo-controlled studies of daily application to the face and balding scalp for two 3-week cycles. J Am Acad Dermatol 2010; 62: 573-81. 